\title{
Fast and Accurate Analysis of Optical Metamaterials Using Surface Integral Equations and the Parallel Multilevel Fast Multipole Algorithm
}

\author{
Özgür Ergül*
}

\begin{abstract}
We present fast and accurate simulations of optical metamaterials using surface integral equations and the multilevel fast multipole algorithm (MLFMA). Problems are formulated with the electric and magnetic current combined-field integral equation and solved iteratively with MLFMA, which is parallelized using the hierarchical strategy on distributed-memory architectures. Realistic metamaterials involving dielectric, perfectly conducting, and plasmonic regions of finite extents are solved rigorously with the developed implementation without any periodicity assumptions.
\end{abstract}

\section{SURFACE INTEGRAL EQUATIONS AND MLFMA FOR OPTICAL META- MATERIALS}

Following their successful implementations at microwave frequencies, it has been desirable to realize nanoscale metamaterials for various applications, such as cloaking, negative refraction, and subwavelength focusing, at optical frequencies. Some examples in the literature for optical metamaterials are long nanowires [1]-[3], fishnet structures [4],[5], nanoscale coaxial waveguides, and metasurfaces. In the literature, computational analysis of these structures is dominated by volume formulations, although surface formulations can provide more efficient solutions since they require discretizations of only interfaces between different regions. Using fast solvers, such as the multilevel fast multipole algorithm (MLFMA), three-dimensional metamaterials can be modeled very accurately without resorting to any periodicity assumptions.

At microwave frequencies, major challenges in metamaterial simulations are ill-conditioned and multi-scale properties of these problems. By employing robust preconditioners [6] and broadband solvers [7], we have been able to analyze reallife metamaterials involving large numbers of subwavelength units discretized with millions of unknowns [8]. At optical frequencies, metamaterial problems are usually better conditioned and

\footnotetext{
*Department of Electrical and Electronics Engineering, Middle East Technical University, Ankara, Turkey, e-mail: ozgur.ergul@eee.metu.edu.tr, tel.: +90312 2104580.

${ }^{\dagger}$ Department of Electrical and Electronics Engineering, Bilkent University, Ankara, Turkey, e-mail: lgurel@ee.bilkent.edu.tr, tel.: +90 3122902096.
}

\author{
Levent Gürel ${ }^{\dagger}$
}

nanoscale metamaterials contain less detailed unit cells compared to their microwave counterparts. But, for realistic simulations (e.g., taking into account the finite extent of realistic structures and fabrication errors), metamaterial problems need to be modeled with large numbers of unknowns. Hence, fast solvers and their parallel implementations are also required at optical frequencies.

It is well known that, depending on the frequency, metals may behave as plasmonic materials with finite negative permittivity values [9]. By carefully selecting correct branches [10], it is relatively straightforward to apply surface integral equations derived for dielectric objects to plasmonics [11],[12]. It is also possible to apply fast solvers, such as MLFMA, for different regions with arbitrary material properties, including negative permittivity and permeability values [13],[14]. On the other hand, metamaterials at optical frequencies are often composite structures involving multiple (plas-

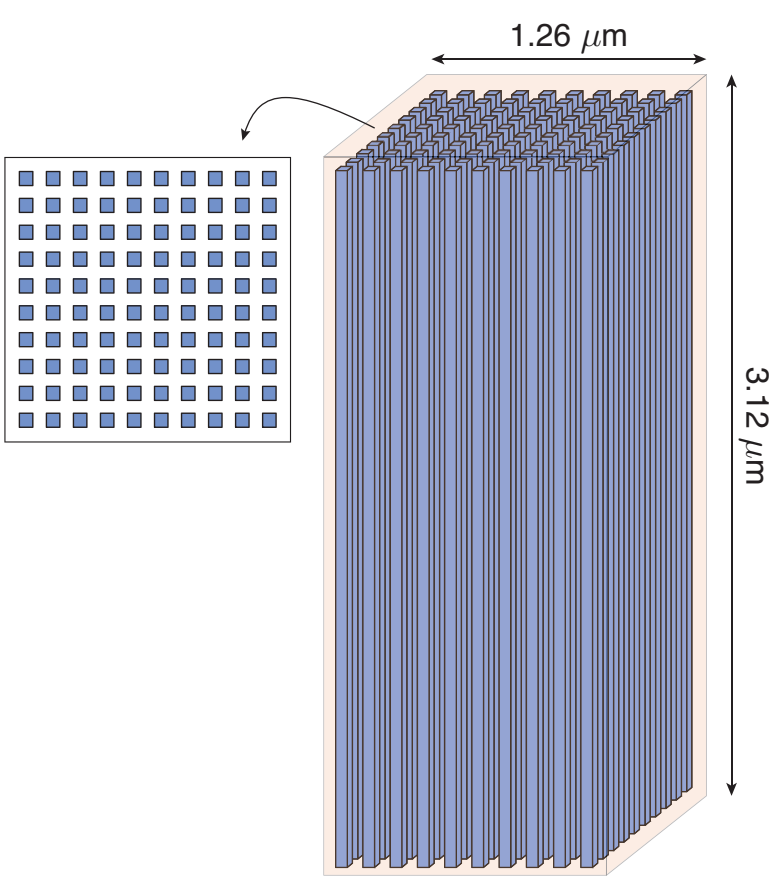

Figure 1: An optical metamaterial involving long metallic rods placed inside a dielectric box. 

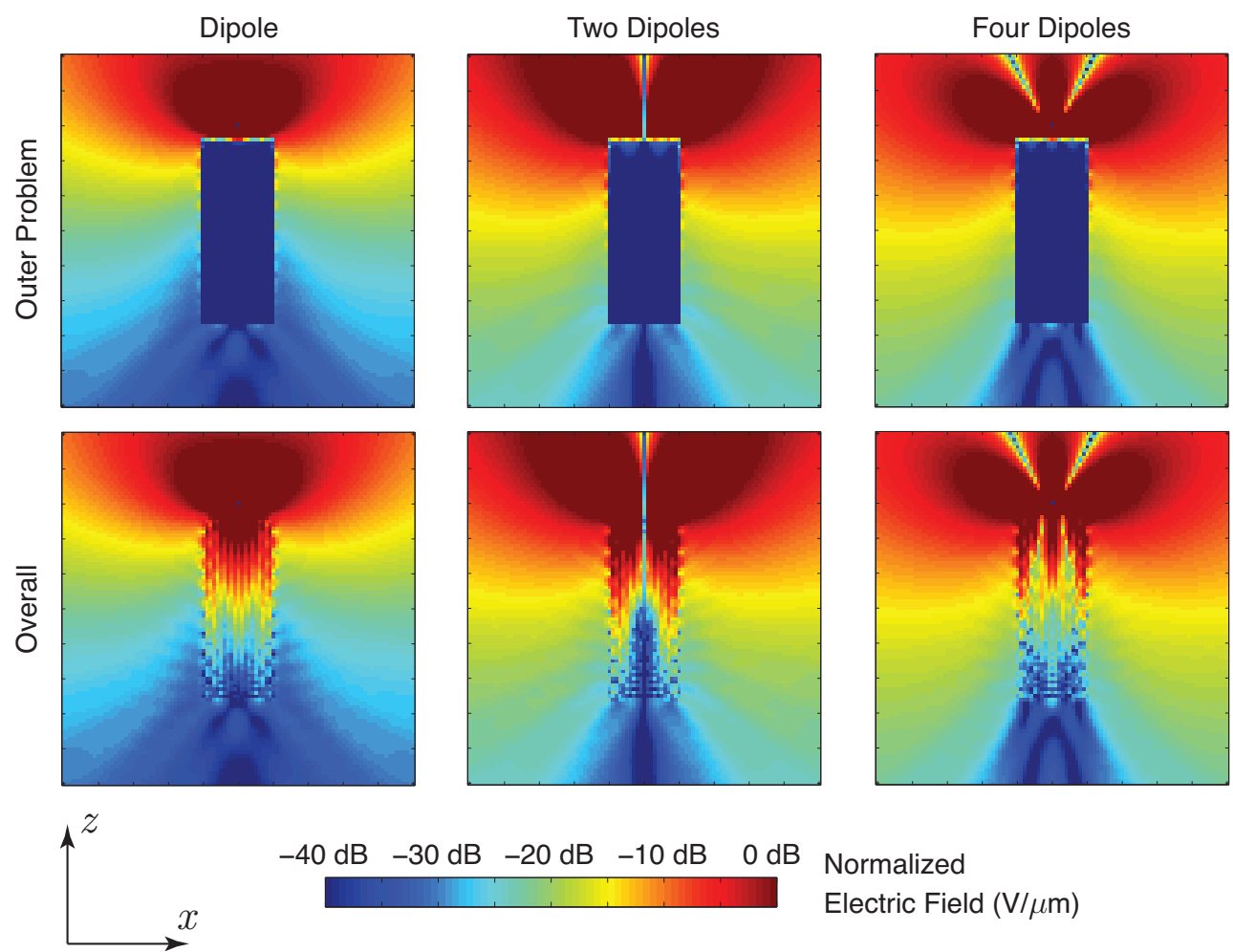

Figure 2: Solutions of electromagnetics problems involving a metamaterial structure depicted in Fig. 1 excited by various dipole configurations. At $417 \mathrm{THz}$, the rods are modeled as perfectly conducting, whereas the box is lossy with a relative permittivity of $9.6+0.4 i$. The total electric field is plotted in the vicinity of the structure.

monic, lossless or lossy dielectric, perfectly conducting) parts. For such composite structures, which lead to tree structures of different sizes and properties, parallelization of MLFMA can be challenging and it requires special techniques, such as the asynchronous parallelization [15].

Recently, we developed hierarchical partitioning strategy for homogeneous dielectric objects with different material properties [16],[17]. This strategy allows for fine-tuning the partitions of subdomains and their samples for efficient parallelizations on distributed-memory architectures. For a given tree structure associated with a medium, numbers of subdomains and field partitions can be adjusted for the best efficiency, considering the number of subdomains (that depends on the surfaces enclosing the medium) and the number of field samples (that depends on the medium parameters). Hence, the hierarchical strategy can also provide efficient parallelization of MLFMA for composite structures.

\section{NUMERICAL SIMULATIONS}

As numerical examples, we consider simulations of optical metamaterials involving long metallic rods. This family of structures is known to provide wide-angle negative refraction for specific po- larizations [1]-[3]. Problems are formulated with the electric and magnetic current combined-field integral equation (JMCFIE) and discretized with the Rao-Wilton-Glisson functions. This combination is known to provide accurate and stable iterative solutions of large-scale objects [18].

Fig. 1 depicts a relatively small metamaterial involving a total of $10 \times 10$ rectangular rods placed inside a dielectric box. The size of each rod is $0.06 \times 0.06 \times 3 \mu \mathrm{m}$ and the size of the overall structure is $1.26 \times 1.26 \times 3.12 \mu \mathrm{m}$. At $417 \mathrm{THz}$, the rods are modeled as perfectly conducting, whereas the box is lossy with a relative permittivity of $9.6+0.4 i$. For numerical solutions, the problem is discretized with 156,516 unknowns. Fig. 2 presents the electric field in the vicinity of the structure on the $z-x$ plane, when it is excited by Hertzian dipoles placed at $0.24 \mu \mathrm{m}$ above the top surface. In the first column, only a $y$-directed dipole is used. In the second column, two dipoles in the $y$ and $-y$ directions are placed with $0.48 \mu \mathrm{m}$ distance between them. Finally, in the third column, three dipoles in the $y$, $-y$, and $y$ directions are placed with $0.24 \mu \mathrm{m}$ intervals. In all cases, the electric field is plotted for the outer equivalent problem (host medium) in addition to the overall field distribution. It can be observed that very small radiation (generally less 


\begin{tabular}{|c|c|c|c|}
\hline Excitation & Iterations & Total Time (hours) & Peak Memory (GB) \\
\hline Two Dipoles & 25 & 8.92 & 495 \\
\hline Four Dipoles & 22 & 8.94 & 495 \\
\hline V-Polarized Beam & 29 & 9.05 & 495 \\
\hline H-Polarized Beam & 29 & 9.00 & 495 \\
\hline
\end{tabular}

Table 1: Solutions of electromagnetics problems involving a large metamaterial structure discretized with $13,224,714$ unknowns.
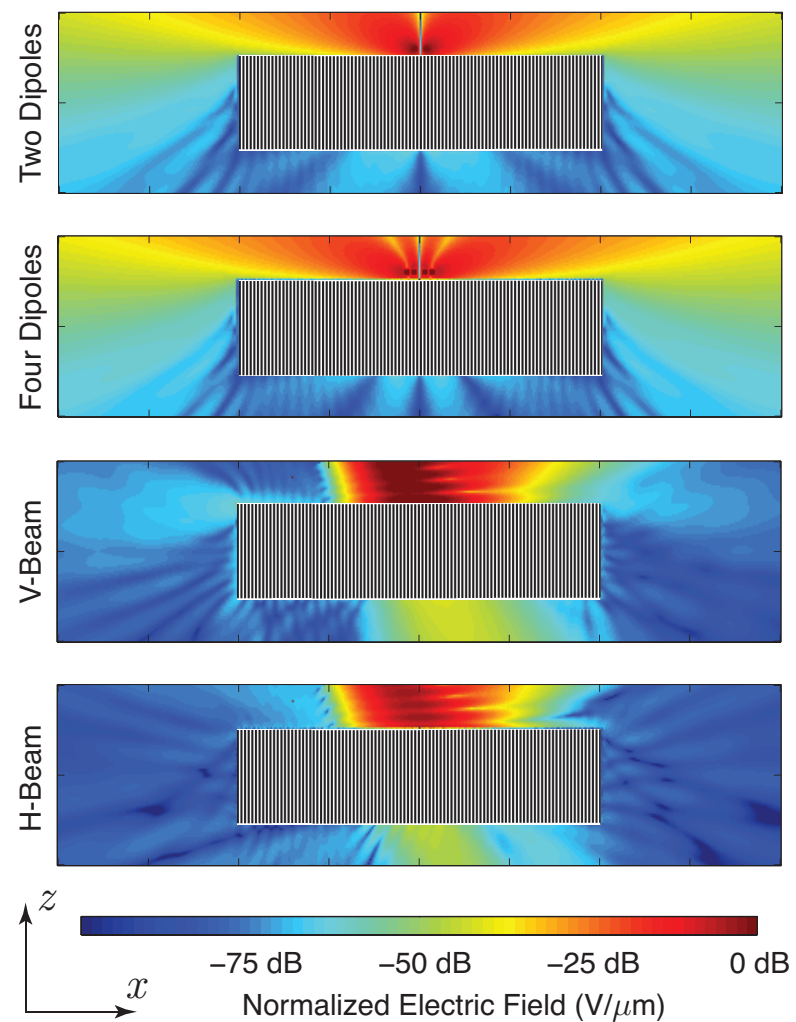

Figure 3: Solutions of electromagnetics problems involving a large metamaterial structure excited by various sources. At $417 \mathrm{THz}$, the rods are modeled as perfectly conducting, whereas the box is lossy with a relative permittivity of $2.4+0.4 i$. The total electric field is plotted in the vicinity of the structure.

than $-40 \mathrm{~dB}$ ) is observed inside the structure for the outer problem, proving the high accuracy of the solutions. In the overall plots, it can be observed that the excitation shapes are transmitted along the structure [3] until the electromagnetic waves decay due to losses.

In order to demonstrate the capabilities of the developed implementation, we consider very large transmission problems involving a total of $101 \times 101$ metallic rods placed inside a dielectric box. The overall dimension of the structure is $12.18 \times 12.18 \times$ $3.12 \mu \mathrm{m}$ and it is discretized with $13,224,714$ unknowns for simulations at $417 \mathrm{THz}$. The rods are again modeled as perfectly conducting, whereas the box is lossy with a relative permittivity of $2.4+0.4 i$.

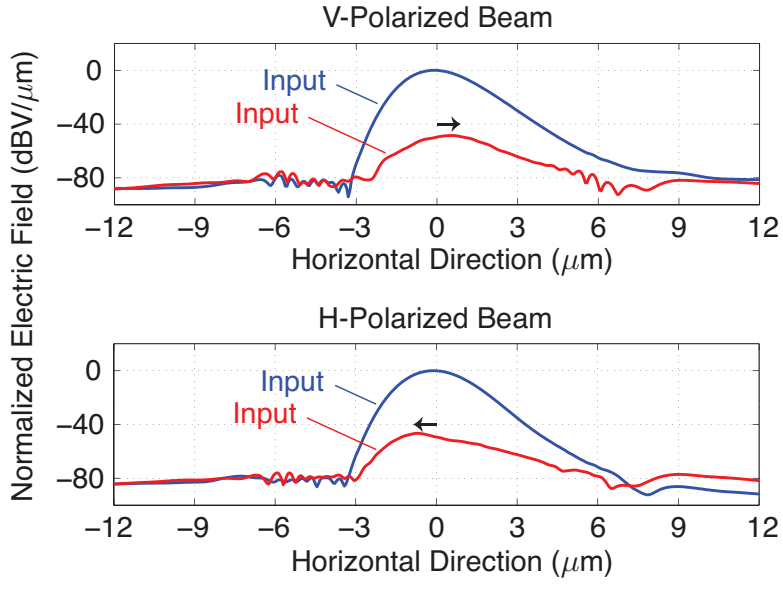

Figure 4: Solutions of electromagnetics problems involving an optical metamaterial (a total of $101 \times 101$ plasmonic rods inside a lossy box) excited by beams. The structure exhibits negative refraction when the beam is H-polarized.

Table 1 lists the number of iterations (for 0.005 residual error), total processing time, and peak memory required for solutions when the structure is excited by various sources, i.e., two different configurations of Hertzian dipoles and two different electromagnetic beams created by complex-sourcepoint dipoles. The problems are solved by MLFMA with two digits of accuracy on 64 cores of a cluster of Intel Xeon Nehalem processors with $2.80 \mathrm{GHz}$ clock rate. As shown in Table 1 , the total time is around 9 hours for each excitation. Fig. 3 depicts the total electric field in the vicinity of the structure, where the transmission properties are investigated for different excitations.

Finally, we demonstrate a negative refraction obtained by long metallic rods. We consider a $101 \times 101$ arrangement of $0.06 \times 0.06 \times 1.8 \mu \mathrm{m}$ rods inside a $12.18 \times 12.18 \times 1.92 \mu \mathrm{m}$ dielectric box. The structure is discretized with 8,216,880 unknowns for simulations at $417 \mathrm{THz}$. The rods are modeled as plasmonic with a relative permittivity of $-8+i$, whereas the box is lossy with a relative permittivity of $1.2+0.1 i$. The structure is illuminated by V-polarized and H-polarized beams. The total processing time, including the setup and two iterative solutions (190 iterations), is less than 14 
hours. Fig. 4 depicts the electric field in the input and output sides of the structure. For the Vpolarized beam, positive refraction occurs and the maximum electric field shifts in the positive direction. For the H-polarized beam, however, the shift is in the negative direction due to the negative refraction. These results are in agreement with theoretical, computational, and measurement results in the literature [1],[2].

\section{Acknowledgments}

This work was supported by the Scientific and Technical Research Council of Turkey (TUBITAK) under Research Grants 110E268 and 111E203 and by contracts from ASELSAN and SSM.

\section{References}

[1] J. Yao, Z. Liu, Y. Liu, Y. Wang, C. Sun, G. Bartal, A. M. Stacy, and X. Zhang, "Optical negative refraction in bulk metamaterials of nanowires," Science, vol. 321, no. 5891, p. 930, Aug. 2008.

[2] Y. Liu, G. Bartal, and X. Zhang, "All-angle negative refraction and imaging in a bulk medium made of metallic nanowires in the visible region," Opt. Express, vol. 16, no. 20, pp. 15439-15448, Sep. 2008.

[3] B. D. F. Casse, W. T. Lu, Y. J. Huang, E. Gultepe, L. Menon, and S. Sridhar, "Superresolution imaging using a three-dimensional metamaterials nanolens," Appl. Phys. Lett., vol. 96, no. 2, pp. 023114-1-023114-3, Jan. 2010.

[4] J. Valentine, S. Zhang, T. Zentgraf, E. UlinAvila, D. A. Genov, G. Bartal, and X. Zhang, "Three-dimensional optical metamaterial with a negative refractive index," Nature, vol. 455, pp. 376-379, Sep. 2008.

[5] S. S. Kruk, D. A. Powell, A. Minovich, D. N. Neshev, and Y. S. Kivshar, "Spatial dispersion of multilayer fishnet metamaterials," Opt. Express, vol. 20, no. 14, pp. 15100-15105, Jun. 2012.

[6] Ö. Ergül, T. Malas, and L. Gürel, "Solutions of large-scale electromagnetics problems using an iterative inner-outer scheme with ordinary and approximate multilevel fast multipole algorithms," Prog. Electromagn. Res., vol. 106, pp. 203-223, 2010.

[7] Ö. Ergül and L. Gürel, "Efficient solutions of metamaterial problems using a low-frequency multilevel fast multipole algorithm," Prog. Electromagn. Res., vol. 108, pp. 81-99, 2010.
[8] L. Gürel, Ö. Ergül, A. Ünal, and T. Malas, "Fast and accurate analysis of large metamaterial structures using the multilevel fast multipole algorithm," Prog. Electromagn. Res., vol. 95, pp. 179-198, 2009.

[9] P. B. Johnson and R. W. Christy, "Optical constants of the noble metals," Phys. Rev. B, vol. 6, no. 12, pp. 4370-4379, Dec. 1972.

[10] M.-F. Wu, X.-X. Liu, A. Alu, and A. E. Yilmaz, "A fast surface integral equation solver for composite structures with metamaterial regions," in Proc. IEEE Antennas and Propagation Soc. Int. Symp., 2011.

[11] J. Rivero, J. M. Taboada, L. Landesa, F. Obelleiro, and I. Garcia-Tunon, "Surface integral equation formulation for the analysis of left-handed metamaterials," Opt. Express, vol. 18, no. 15, pp. 15876-15886, Jul. 2010.

[12] Ö. Ergül, "Analysis of composite nanoparticles with surface integral equations and the multilevel fast multipole algorithm," J. Opt., vol. 14, no. 6, pp. 062701-1-062701-4, Jun. 2012.

[13] Ö. Ergül, "Fast and accurate analysis of homogenized metamaterials with the surface integral equations and the multilevel fast multipole algorithm," IEEE Antennas Wireless Propag. Lett., vol. 10, pp. 1286-1289, 2011.

[14] M. G. Araujo, J. M. Taboada, J. Rivero, D. M. Solis, and F. Obelleiro, "Solution of largescale plasmonic problems with the multilevel fast multipole algorithm," Opt. Lett., vol. 37, no. 3, pp. 416-418, Feb. 2012.

[15] J. Fostier and F. Olyslager, "An asynchronous parallel MLFMA for scattering at multiple dielectric objects," IEEE Trans. Antennas Propag., vol. 56, no. 8, pp. 2346-2355, Aug. 2008.

[16] Ö. Ergül, "Parallel implementation of MLFMA for homogeneous objects with various material properties," Prog. Electromagn. Res., vol. 121, pp. 505-520, 2011.

[17] Ö. Ergül, "Solutions of large-scale electromagnetics problems involving dielectric objects with the parallel multilevel fast multipole algorithm," J. Opt. Soc. Am. A., vol. 28, no. 11, pp. 2261-2268, Nov. 2011.

[18] Ö. Ergül and L. Gürel, "Comparison of integral-equation formulations for the fast and accurate solution of scattering problems involving dielectric objects with the multilevel fast multipole algorithm," IEEE Trans. Antennas Propag., vol. 57, no. 1, pp. 176-187, Jan. 2009. 